\title{
Avaliação do comportamento ingestivo de vacas leiteiras em pastejo de Brachiaria brizantha recebendo diferentes teores de concentrado na dieta
}

\author{
Evaluation of dairy cows intake behavior grazing Brachiaria \\ brizantha fed different levels of concentrate at diet
}

\author{
Fabrício Bacelar Lima Mendes ${ }^{1 *}$; Fabiano Ferreira da Silva ${ }^{2}$; Robério Rodrigues Silva ${ }^{2}$; \\ Gleidson Giordano Pinto de Carvalho ${ }^{3}$; Elisangela Oliveira Cardoso ${ }^{1}$; \\ Aires Lima Rocha Neto ${ }^{4}$ Julinessa Silva Oliveira ${ }^{1}$; Lucas Teixeira Costa ${ }^{5}$; \\ Hermógenes Almeida de Santana Júnior ${ }^{6}$; Alyson Andrade Pinheiro ${ }^{7}$
}

\section{Resumo}

\begin{abstract}
Objetivou-se avaliar o comportamento ingestivo de vacas leiteiras em pastagem de Brachiaria brizantha submetidas a diferentes teores de suplementação concentrada na dieta. Utilizaram-se 16 vacas lactantes $1 / 2$ Holandês $\times 1 / 2$ Zebu, com média de $80 \pm 10$, 14 dias de lactação e peso corporal de 454,7 $\pm 54,51 \mathrm{~kg}$. Os animais foram distribuídos em quatro tratamentos, utilizando-se um quadrado latino 4 $\times 4$. O comportamento foi avaliado a cada dez minutos, durante 24 horas no $16^{\circ}$ dia de cada período. Utilizou-se a análise de variância e regressão a 0,05 de probabilidade. Os tempos despendidos em pastejo, ruminação e outras atividades; os números de pastejo, ruminação, de bocados por dia e de mastigações merícicas por bolo; os tempos de pastejo, alimentação total e mastigação total; a taxa de bocado; o tempo de deglutição; o tempo por bolo ruminado; a velocidade de mastigação e a eficiência de alimentação para FDN não sofreram alterações com o aumento nos teores de concentrado na dieta. $\mathrm{O}$ tempo de cocho, o período de cocho, o tamanho de bocado, o tempo por mastigação de bolo, a eficiência de alimentação para MS, CNF, NDT e a eficiência de ruminação cresceram linearmente. O período de ruminação, o número de bocados por deglutição, o número de bolos ruminados por dia e o número de mastigações merícicas por dia decresceram linearmente com o aumento dos teores de concentrado na dieta. Houve efeito quadrático do teor de concentrado sobre o número de períodos em outras atividades e sobre o tempo em outras atividades. A suplementação com até $33,8 \%$ de concentrado na dieta de vacas em pastejo não promovem alterações nas atividades de pastejo e ruminação nem o tempo gasto em outras atividades, porém aumenta o tempo que os animais permanecem no cocho. $\mathrm{O}$ aumento no teor de concentrado da dieta melhora as eficiências de alimentação e ruminação.
\end{abstract}

Palavras-chave: Bovino, consumo, pastagem, produção, ruminante

\footnotetext{
${ }^{1}$ Discentes do Curso de Doutorado em Produção Animal, Universidade Estadual do Sudoeste da Bahia, UESB, Itapetinga, BA. E-mail: fabricio.bacelar@hotmail.com; eocardoso@hotmail.com; julinessa1@hotmail.com

2 Profs. Drs. do Dept ${ }^{\circ}$ de Zootecnia da Universidade Estadual do Sudoeste da Bahia, UESB, Itapetinga, BA. Bolsista de produtividade do CNPq.E-mail: ffsilva@pq.cnpq.br; rrsilva.uesb@hotmail.com

${ }^{3}$ Prof. Dr. do Dept ${ }^{\mathrm{o}}$ de Zootecnia da Universidade Federal da Bahia, UFBA, Salvador, BA. Bolsista de Produtividade do CNPq. E-mail: geidsongiordano@yahoo.com.br

${ }^{4}$ Prof. Dr. do Instituto Federal Baiano, IFBaiano, Santa Inês, BA. E-mail: anzol22@hotmail.com

${ }^{5}$ Dr. em Zootecnia, UESB, Itapetinga, BA. E-mail: lucastc80@hotmail.com

${ }^{6}$ Prof. Dr. do Dept ${ }^{\circ}$ de Zootecnia da Universidade Estadual do Piauí, UESPI, Corrente, PI. E-mail: hsantanajunior@hotmail.com

${ }^{7}$ Dr. em Zootecnia, Pesquisador da Agencia Goiana de Assistência Técnica, Extensão Rural e Pesquisa Agropecuária, EMATER, Goiânia, GO. E-mail: jagualyson@bol.com.br

* Autor para correspondência
} 


\begin{abstract}
The objective with this research was to evaluate the dairy cows intake behavior grazing Brachiaria brizantha submitted to different levels of concentrate supplementation in the diet. Used 16 lactating cows $1 / 2$ Holstein $\times 1 / 2$ Zebu, with an average of $80 \pm 10.14$ days of lactation and body weight of 454.7 $\pm 54.51 \mathrm{~kg}$. The animals were distributed in four treatments, using a $4 \times 4$ Latin square. The evaluation of behavior was done every ten minutes, during 24 hours on the 16th day of period. We used analysis of variance and regression probability of 0.05 . The time spent grazing, ruminating and other activities, the numbers of grazing, ruminating, of bites per day and chews per cake, the grazing times, total power and total chewing, bit rate, time to swallow; time per bolus ruminated; chewing speed and power efficiency for NDF were not altered by increased levels of concentrate. The time of trough, the trough period, the bit size, the chews time for bolus, feeding efficiency for DM, NFC, and TDN and rumination efficiency linearly increased. Periods of rumination, deglutition bits, number of rumination chews per day, decreased linearly with increasing levels of concentrate. Significant quadratic effect of concentrate level on the number of periods in other activities and over time on other activities. Supplementation with $33.8 \%$ concentrate in the diet of cows grazing not promote changes in the activity of grazing, ruminating and other activities, but increases the time of trough. The increase in concentrate diet improves the efficiencies of feeding and rumination.
\end{abstract}

Key words: Cattle, intake, grazing, production, ruminant

\section{Introdução}

Opastejoéum processo de elevada complexidade, uma vez que envolve, simultaneamente, características do animal herbívoro e do alimento presente em seu ambiente (PRACHE; GORDON; ROOK, 1998), cujas interações refletirão na capacidade de aquisição de nutrientes pelo animal e sobre o impacto que esse processo incorrerá sobre a vegetação. De acordo com Rutter et al. (2002), o conhecimento dos padrões de comportamento da escolha, localização e ingestão do pasto pelo animal é de fundamental importância quando se pretende estabelecer práticas de manejo.

O estudo do comportamento ingestivo pode nortear a adequação do sistema de alimentação para o aumento da produtividade e a garantia de alta produção, melhor estado sanitário e longevidade aos animais (FISCHER et al., 2002). Nesse sentido, tem-se investido em pesquisas que permitam entender o comportamento ingestivo de animais ruminantes, buscando sempre o manejo nutricional adequado desses animais (SILVA et al., 2004).

A avaliação do comportamento ingestivo de vacas mestiças lactantes em pastejo recebendo suplementação é essencial para o manejo adequado desses animais, uma vez que a suplementação com concentrado não deve ser utilizada com o objetivo de substituir o pasto, mas sim complementá-lo. No entanto, nem sempre esse objetivo é alcançado, pois muito comumente, quando se aplica a suplementação com elevado teor de suplemento, os animais substituem parte da forragem que deveria ser consumida pelo concentrado.

A suplementação da dieta para vacas mestiças em lactação deve ser eficiente, uma vez que vacas dessa categoria passam parte do dia no curral, para que sejam ordenhadas. Dessa forma, os horários em que os animais se encontram no curral são os melhores momentos para que possa fornecer o concentrado, não alterando o horário de pastejo dos animais, visando a resposta positiva e o aumento na produtividade do rebanho. De acordo com Mendes Neto et al. (2007), uma simples modificação no horário ou na frequência de fornecimento do concentrado pode causar mudança nos hábitos alimentares de bovinos, podendo, com isso, influenciar na sua produtividade.

Objetivou-se com este estudo avaliar o comportamento ingestivo de vacas leiteiras em pastagem de Brachiaria brizantha submetidas a 
diferentes níveis de suplementação concentrada na dieta.

\section{Material e Métodos}

O experimento foi conduzido na Fazenda Paulistinha, situada em Macarani, BA, localizada a $15^{\circ} 33^{\prime} 46^{\prime \prime}$ de latitude Sul, $40^{\circ} 25^{\prime} 38^{\prime \prime}$ de longitude Oeste, com altitude média de $315 \mathrm{~m}$, possuindo clima tropical AW, segundo a classificação de
Köppen-Geiger (Tabela 1). O trabalho de campo foi realizado entre os meses de fevereiro a abril de 2009 em uma área 42 hectares, dividida em 8 piquetes de aproximadamente 5,3 hectares cada formados por Brachiaria brizantha cultivar Marandu e contendo bebedouros. Utilizou-se a lotação rotativa, com controle de entrada dos animais nos piquetes de acordo com a altura do pasto, considerando 40 cm para a entrada e $20 \mathrm{~cm}$ para a saída. A taxa de lotação foi de 1,21 UA/ha.

Tabela 1. Médias das temperaturas máximas (Tmax) e mínimas (Tmin) e precipitação pluviométrica total, por mês, observadas durante a fase experimental.

\begin{tabular}{lccc}
\hline \multirow{2}{*}{$\mathrm{T}\left({ }^{\circ} \mathrm{C}\right)$} & & Mês & \\
\cline { 2 - 4 } & Fevereiro & Março & Abril \\
\hline Tmax $\left({ }^{\circ} \mathrm{C}\right)$ & 34,0 & 37,0 & 36,0 \\
Tmin $\left({ }^{\circ} \mathrm{C}\right)$ & 20,0 & 20,0 & 21,0 \\
Média & 28,0 & 28,9 & 27,6 \\
Precipitação $(\mathrm{mm})$ & 5,6 & 83,4 & 112,4 \\
\hline
\end{tabular}

Fonte: Universidade Estadual do Sudoeste da Bahia (UESB).

Foram utilizadas 16 vacas lactantes Holandês x Zebu com $80 \pm 10,14$ dias de lactação e peso corporal médio de 454,7 $\pm 54,51 \mathrm{~kg}$, distribuídas em quatro tratamentos, em delineamento experimental de quadrado latino $(4 \times 4)$. Os animais receberam suplementação com concentrado (Tabela 2) para atender às exigências de mantença e produção de 7, 10, 13 e $16 \mathrm{~kg}$ de leite. dia $^{-1}$, de acordo com o (NRC, 2001).

Tabela 2. Proporção dos ingredientes nos concentrados, com base na matéria natural.

\begin{tabular}{lccc}
\hline Ingrediente & \multicolumn{3}{c}{ Concentrado na dieta (\%) } \\
\cline { 2 - 4 } Calcário calcítico (\%) & $\mathrm{C} 16,4$ & $\mathrm{C} 23,3$ & $\mathrm{C} 33,8$ \\
Farelo de soja (\%) & 0,0 & 0,48 & 0,81 \\
Fubá de milho (\%) & 56,72 & 38,42 & 29,74 \\
Ureia (\%) & 36,33 & 57,16 & 66,29 \\
Sal mineral $^{1}(\%)$ & 0,05 & 0,28 & 0,78 \\
\hline
\end{tabular}

${ }^{1}$ Composição: cálcio, 18,5\%; fósforo, 9\%; magnésio, 0,4\%; enxofre, 1\%; sódio, 11,7\%; selênio, 30 ppm; cobre, 1500 ppm; zinco, 4000 ppm; manganês, 1200 ppm; iodo, 150 ppm; cobalto, 150 ppm.

Fonte: Elaboração dos autores.

Como tratamentos, consideraram-se os teores de concentrado incluídos na dieta: C0 - Dieta com $0 \%$ de inclusão de concentrado na matéria seca natural (controle); C16,4 - Dieta com 16,4\% na matéria seca natural (MSN); C23,3 - Dieta com 23,3\% de inclusão de concentrado na MSN; C33,8 - Dieta com 
$33,8 \%$ de inclusão de concentrado na matéria seca natural (MSN). A relação volumoso:concentrado nas dietas (Tabela 3) foi de 100:0; 83,6:16,4; $76,7: 23,3$ e $66,2: 33,8$, com base na matéria seca natural (MSN).

O experimento teve duração de 68 dias, divididos em quatro períodos de 17 dias. Desses, 10 dias foram destinados de adaptação dos animais às dietas e sete, de coleta de dados. As vacas retornavam dos piquetes para o curral às quatro horas e trinta minutos, para a realização da primeira ordenha, sendo a segunda ordenha iniciada por volta das $16 \mathrm{~h}$. A ordenha era realizada de forma manual, com o bezerro ao pé e sempre pelo mesmo ordenhador. O suplemento era fornecido aos animais logo após as ordenhas, em baias individuais providas de cochos com disponibilidade de $150 \mathrm{~cm}$ lineares para cada animal.

Tabela 3. Composição química do volumoso e dos concentrados em função do tratamento.

\begin{tabular}{lcccc}
\hline \multirow{2}{*}{ Nutrientes } & \multirow{2}{*}{ Volumoso } & \multicolumn{3}{c}{ Concentrado na dieta (\%) } \\
\cline { 3 - 5 } & & $\mathrm{C} 16,4$ & $\mathrm{C} 23,3$ & $\mathrm{C} 33,8$ \\
\hline MS \% & 23,3 & 84,8 & 84,6 & 84,3 \\
MO $^{1}$ & 91,7 & 89,9 & 93,6 & 93,9 \\
PB $^{1}$ & 8,2 & 34,8 & 26,9 & 25,5 \\
EE $^{1}$ & 4,0 & 2,8 & 3,38 & 3,8 \\
FDNcp $^{1}$ & 63,8 & 29,8 & 33,3 & 31,9 \\
FDA $^{1}$ & 42,6 & 8,7 & 8,9 & 9,7 \\
CNF $^{1}$ & 15,7 & 22,5 & 30,1 & 32,7 \\
HEM $^{1}$ & 30,8 & 4,8 & 6,6 & 6,0 \\
CEL $^{1}$ & 35,0 & 5,2 & 6,7 & 5,9 \\
LIG $^{1}$ & 5,2 & 1,2 & 2,8 & 2,2 \\
MM $^{1}$ & 8,2 & 10,0 & 6,3 & 6,0 \\
\hline
\end{tabular}

${ }^{1} \mathrm{Em} \%$ da MS.

Fonte: Elaboração dos autores.

O pasto foi avaliado diariamente utilizando-se o método de McMeniman (1997), Wilm, Costello e Klipple (1994), Gardner (1986) e Campbell (1966) para a coleta e determinação da biomassa residual diária (BRD), da biomassa de forragem (disponibilidade) e da taxa de acúmulo diário. A oferta de forragem (OF) foi calculada de acordo com a seguinte fórmula: $\mathrm{OF}=\{(\mathrm{BRD} *$ área $+\mathrm{TAD} *$ área $) /$ $\left.\mathrm{PC}_{\text {total }}\right\} * 100$, em que: $\mathrm{OF}=$ oferta de forragem, em $\mathrm{kg} \mathrm{MS} / 100 \mathrm{~kg} \mathrm{PV} /$ dia; BRD = biomassa residual total, em $\mathrm{kg}$ de $\mathrm{MS} / \mathrm{ha} /$ dia; TAD = taxa de acúmulo diário, em $\mathrm{kg} \mathrm{MS} / \mathrm{ha} /$ dia; e PC = peso corporal total dos animais, em $\mathrm{kg} / \mathrm{ha}$.

A matéria seca potencialmente digestível (MSpd) do pasto foi determinada conforme metodologia descrita por Paulino et al. (2006), pela seguinte equação: $\mathrm{MSpd}=0,98 *(100-\% \mathrm{FDN})+$ (\%FDN-\%FNDi). A estimativa de produção fecal foi estimada utilizando-se o óxido crômico como indicador externo, fornecido diariamente durante 12 dias, ou seja, sete dias para adaptação e cinco para coleta. O cálculo foi elaborado com base na razão entre a quantidade do indicador fornecido e sua concentração nas fezes, segundo a equação: EF = (Crfornecido/Crfezes) x 100, em que: CrFornecido $=$ quantidade de cromo fornecido $(\mathrm{g})$ e CrFezes = concentração do indicador nas fezes $(\%)$. Para estimativa do consumo voluntário de volumoso, foi utilizado o indicador interno fibra em detergente neutro indigestível (FDNi), obtido após incubação ruminal por 240 horas (Casali et al., 2008), de 0,5 g de amostras de alimentos, sobras e fezes, utilizando- 
se sacos confeccionados com tecido-não-tecido (TNT) gramatura $100(100$ g.m² $), 5$ x $5 \mathrm{~cm}$. O material remanescente da incubação foi submetido à extração com detergente neutro, para determinação da fibra em detergente neutro indigestível (FDNi).

O consumo de matéria seca (MS) foi obtido com o uso da seguinte equação: $\mathrm{CMS}=\left\{\left[\left(\mathrm{PF}{ }^{*} \mathrm{CIFZ}\right)\right.\right.$ IS]/CIFR $\}$ + CMSS, em que CMS é o consumo de matéria seca (kg/dia); PF, a produção fecal ( $\mathrm{kg} /$ dia); CIFZ, a concentração do indicador presente nas fezes $(\mathrm{kg} / \mathrm{kg})$; IS, o indicador presente no suplemento $(\mathrm{kg} /$ dia); CIFR, a concentração do indicador presente na forragem $(\mathrm{kg} / \mathrm{kg})$; e o CMSS, o consumo de matéria seca do suplemento ( $\mathrm{kg} / \mathrm{dia})$.

Amostras dos alimentos, assim como das fezes, foram coletadas acondicionadas em sacos plásticos e armazenadas em freezer a $-10^{\circ} \mathrm{C}$, para posteriores análises, realizadas nos laboratórios da UESB. Amostras dos volumosos, do concentrado e das fezes de cada animal foram pré-secas em estufa de ventilação forçada a $60{ }^{\circ} \mathrm{C}$ e moídas em moinho de faca (peneira com crivos de $1 \mathrm{~mm}$ ) para análises químicas. As análises de matéria seca (MS), matéria orgânica (MO), proteína bruta $(\mathrm{PB})$, extrato etéreo (EE), fibra em detergente neutro (FDN), fibra em detergente ácido (FDA), carboidratos não-fibrosos (CNF), celulose, hemicelulose, lignina e matéria mineral (MM) foram realizadas seguindo os procedimentos descritos por Silva e Queiroz (2002). O teor de fibra em detergente neutro corrigido para cinzas e proteínas foi determinado segundo recomendações de Mertens (2002). Os teores de carboidratos não-fibrosos corrigidos para cinzas e proteína $\left(\mathrm{CNF}_{\mathrm{CP}}\right)$ foram calculados como proposto por Hall (2003): $\mathrm{CNF}_{\mathrm{CP}}=(100-\% \mathrm{FDNcp}-\% \mathrm{~PB}-$ $\%$ EE - \%cinzas).

A avaliação do comportamento foi realizada no $16^{\circ}$ dia de cada período experimental, totalizando quatro avaliações, realizadas a cada dez minutos, conforme descrito por Silva et al. (2008), por um período de 24 horas, visando identificar o tempo destinado pelos animais ao pastejo, à ruminação, à alimentação no cocho e em outras atividades. Os animais foram avaliados visualmente, por dois observadores treinados, utilizando-se na observação noturna lanternas para a coleta dos dados.

Os animais foram identificados por fitas coloridas amarradas no pescoço e também por brinco na orelha. As variáveis comportamentais estudadas foram: tempo de pastejo (PAS $\mathrm{min} /$ dia), tempo de ruminação (RUM min/dia), tempo de alimentação no cocho (COC min/dia) e tempo em outras atividades (OUT min/dia). As atividades comportamentais foram consideradas mutuamente excludentes, conforme definição de Pardo et al. (2003).

O tempo gasto pelos animais na seleção e apreensão da forragem, incluindo os curtos espaços de tempo utilizados no deslocamento para a seleção da forragem, foi considerado tempo de pastejo (HANCOCK, 1953). O tempo de ruminação correspondeu aos processos de regurgitação, remastigação, reinsalivação e redeglutição. O tempo de alimentação no cocho foi o tempo despendido pelo animal no consumo de suplemento, enquanto o tempo gasto com outras atividades (descanso, consumo de água, interações etc) foi composto de todas as atividades, com exceção das citadas acima.

Os tempos de alimentação total (TAT (min)) e mastigação total (TMT (min)) foram determinados pelas equações abaixo: TAT $=$ PAS + COC, em que: PAS (minutos) $=$ tempo de pastejo; COC (minutos) $=$ tempo de alimentação no cocho; TMT $=$ PAS + RUM + COC, em que: PAS (minutos) $=$ tempo de pastejo; RUM (minutos) = tempo de ruminação; COC (minutos) $=$ tempo de alimentação no cocho. A discretização das séries temporais foi realizada diretamente nas planilhas de coleta de dados, com a contagem dos períodos discretos de pastejo, ruminação, outras atividades e alimentação no cocho. A duração média de cada um dos períodos discretos foi obtida pela divisão dos tempos diários de cada uma das atividades pelo número de períodos discretos da mesma atividade. 
As observações para determinar as mastigações merícicas e o tempo despendido na ruminação de cada bolo foram realizadas em dois períodos diferentes do dia (de 10 às $12 \mathrm{~h}$; de 14 às 16h), seguindo metodologia de Bürger et al. (2000). A taxa de bocados (TxB boc/s) dos animais de cada tratamento foi estimada por meio do tempo gasto pelo animal para realizar 20 bocados (Hodgson, 1982). Para o cálculo da massa de bocado em MS (MaB gMO), dividiu-se o consumo diário de matéria orgânica da forragem pelo total de bocados diários (JAMIESON; HODGSON, 1979). Foram coletados o número de bocados e o tempo por deglutição. O número de observações foi registrado em seis ocasiões durante o dia, conforme Baggio et al. (2009). As variáveis número de bolo ruminado por dia $\left(B O L \mathrm{n}^{\circ}\right.$ ), velocidade de mastigação (VeM $\mathrm{n} \%$ ), tempo por mastigação merícica (TeM) e mastigações merícicas por dia (MMnd) foram calculadas pelas equações abaixo: $\mathrm{BOL}=\mathrm{RUM} /$ TBo, em que: BOL (n/dia); RUM (s/dia) - tempo de ruminação; TBo (segundos,) - tempo por bolo ruminado; VeM = MMB / TBo, em que: VeM (s); MMB - número de mastigações merícicas por bolo; TBo (s) - tempo por bolo ruminado; TeM = TBo / MMB, em que: TeM (s); TBo (s) - tempo por bolo ruminado; MMB - número de mastigações merícicas por bolo; $\mathrm{MMnd}=\mathrm{BOL} * \mathrm{MMB}$, em que: MMnd (n/dia); BOL - número de bolos ruminados por dia; MMB - número de mastigações merícicas por bolo.

A eficiência de alimentação (EA) e a eficiência de ruminação (ER) para MS, FDN, CNF e NDT foram obtidas segundo metodologia descrita por Bürger et al. (2000).

Para análise dos dados coletados no experimento, foi utilizados o Sistema de Análises Estatísticas e Genéticas - SAEG (RIBEIRO JÚNIOR, 2001). Os modelos estatísticos foram escolhidos de acordo com a significância dos coeficientes de regressão, utilizando-se o teste " $\mathrm{f}$ " a $5 \%$, e de determinação $\left(\mathrm{r}^{2}\right)$.

\section{Resultados e Discussão}

O consumo de matéria seca total aumentou $(\mathrm{P}<0,05)$ com a inclusão dos teores de concentrado na dieta das vacas em lactação e esse aumento pode ser explicado pelo efeito associativo da adição de alimento concentrado sobre o consumo de volumoso pastejado (Tabela 4).

Tabela 4. Consumos de matéria seca total (CMST), matéria seca do pasto (CMSP), e fibra em detergente neutro total (CFDNT), disponibilidade de matéria seca (DPMS) e oferta de forragem (OFF) nos períodos experimentais com suas respectivas equações de regressão (ER), coeficientes de determinação $\left(\mathrm{R}^{2}\right)$, e coeficiente de variação $(\mathrm{CV})$.

\begin{tabular}{|c|c|c|c|c|c|c|c|c|}
\hline \multirow{2}{*}{ Item } & \multicolumn{5}{|c|}{ Concentrado na dieta (\%) } & \multirow{2}{*}{ ER } & \multirow{2}{*}{$\mathrm{R}^{2}$} & \multirow{2}{*}{$\mathrm{CV}$} \\
\hline & $\mathrm{C} 0$ & $\mathrm{C} 16,4$ & $\mathrm{C} 23,3$ & & & & & \\
\hline CMST (kg/dia) & 10,53 & 12,41 & 13,96 & & & 1 & 0,98 & 10,9 \\
\hline CMSP (kg/dia) & 10,53 & 10,43 & 10,21 & & & $\hat{Y}=10,27$ & ---- & 13,9 \\
\hline CFDNT (kg/dia) & 6,72 & 7,25 & 7,76 & & & 2 & 0,98 & 12,2 \\
\hline \multirow{2}{*}{ Item } & & \multicolumn{6}{|c|}{ Período Experimental } & \multirow{2}{*}{ Média } \\
\hline & & $1^{\circ}$ & & & $3^{\circ}$ & & & \\
\hline \multicolumn{2}{|l|}{ DPMS (kg/ha) } & 2,842 & \multicolumn{2}{|c|}{2,119} & 2,232 & \multicolumn{2}{|c|}{3,412} & 2,651 \\
\hline \multicolumn{2}{|l|}{ MSpd (kg/ha) } & 472,21 & \multicolumn{2}{|c|}{351,75} & 379,51 & \multicolumn{2}{|c|}{566,39} & 440,21 \\
\hline \multicolumn{2}{|c|}{ OFF kg MS/100 kg PV } & 2,627 & \multicolumn{2}{|c|}{1,959} & 2,063 & \multicolumn{2}{|c|}{3,154} & 2,450 \\
\hline
\end{tabular}

${ }^{1} \hat{Y}=10,343478+0,151341 X ;{ }^{2} \hat{Y}=6,674919+0,043076 X$.

Fonte: Elaboração dos autores. 
Não foi observada diferença, no entanto, no consumo de matéria seca do pasto com o aumento da quantidade de concentrado fornecida, fato que pode ser considerado positivo, pois indica que não houve substituição da ingestão de forragem pelo concentrado. Esse resultado está em discordância com relatos de Moreno (2002), que, avaliando o efeito da suplementação com farelo de milho sobre o desenvolvimento de novilhas mestiças em pastagens de azevém, observou que a suplementação energética esteve normalmente associada à redução no consumo de forragem. Os resultados obtidos neste estudo divergem também dos reportados por Sousa et al. (2008), que forneceram suplemento concentrado (4, 6 e $8 \mathrm{~kg}$ /animal) a vacas mestiças em pastagens de Brachiaria brizantha, Brachiaria decumbes e Brachiaria ruzizienses e notaram substituição parcial do alimento forrageiro pelo concentrado.

O consumo de fibra em detergente neutro seguiu a mesma tendência do consumo de matéria seca total, com efeito linear crescente de acordo com os teores de concentrado adicionados à dieta. Sousa et al. (2008) também obtiveram resultados semelhantes em pesquisa com vacas mestiças em pastagem de Brachiaria brizantha, Brachiaria decumbens e Brachiaria ruziziensis recebendo suplementação com 4,6 e $8 \mathrm{~kg}$ de concentrado.

Em sistemas de produção que utilizam suplemento concentrado para animais criados em pastos tropicas, é de fundamental importância a interação animal $\times$ suplemento $\times$ planta. Dessa forma, a disponibilidade, a oferta e a qualidade da forragem do pasto podem interferir de forma significativa no consumo dos nutrientes, determinando o sucesso ou não do sistema. Em revisão de literatura, Silva et al. (2009) afirmaram que é necessário garantir o fornecimento de pelo menos $4.500 \mathrm{~kg}$ de MS total.ha- ${ }^{1}$ para permitir seletividade e desempenho individuais satisfatórios sem comprometer a produção por área. Neste estudo, os resultados de disponibilidade de forragem correspondem a $59 \%$ do sugerido por Silva et al. (2009); a 72,5\% do encontrado por Silva (2008), em estudo sobre suplementação para novilhos em terminação no sudoeste da Bahia; e 25\% superior ao citado por Minson (1990) como valor mínimo proposto pelo autor, podendo assim causar interferência no consumo. A baixa disponibilidade de forragem, assim como a oferta encontrada neste estudo, pode ser consquência dos baixos índices pluviométricos nos meses que antecederam o período experimental.

Os teores de concentrado não influenciaram $(\mathrm{P}>0,05)$ os tempos despendidos em pastejo, ruminação e outras atividades (Tabela 5), fato que pode ser atribuído à similaridade do consumo de MS do pasto. Esses resultados contrariam os relatos de Fischer et al. (2002), que verificaram redução linear dos tempos de pastejo em função do aumento dos teores de suplementação.

Tabela 5. Médias e equações de regressão dos tempos (min) despendidos em pastejo, no cocho, em ruminação e outras atividades em função dos teores de concentrado da dieta com suas respectivas equações de regressão (ER), coeficientes de determinação $\left(\mathrm{R}^{2}\right)$, e coeficiente de variação $(\mathrm{CV})$.

\begin{tabular}{|c|c|c|c|c|c|c|c|}
\hline \multirow{2}{*}{ Item } & \multicolumn{4}{|c|}{ Concentrado na dieta $(\%)$} & \multirow{2}{*}{ ER } & \multirow{2}{*}{$\mathrm{R}^{2}$} & \multirow{2}{*}{$\mathrm{CV}$} \\
\hline & $\mathrm{C} 0$ & $\mathrm{C} 16,4$ & $\mathrm{C} 23,3$ & $\mathrm{C} 33,8$ & & & \\
\hline Pas (min/dia) & 586,25 & 628,75 & 571,88 & 590,63 & $\hat{\mathrm{Y}}=594,38$ & - & 14,15 \\
\hline $\operatorname{Coc}(\mathrm{min} / \mathrm{dia})$ & 0,00 & 25,63 & 38,3 & 45,0 & 1 & 0,97 & 19,67 \\
\hline $\operatorname{Rum}(\min / \mathrm{dia})$ & 521,88 & 461,25 & 514,38 & 465,00 & $\hat{Y}=490,63$ & - & 14,18 \\
\hline Out (min/dia) & 331,88 & 324,38 & 315,63 & 341,88 & $\hat{\mathrm{Y}}=328,44$ & - & 18,37 \\
\hline
\end{tabular}

${ }^{1} \hat{Y}=1,886683+1,376915 \mathrm{X}$

Fonte: Elaboração dos autores. 
O tempo que o animal passou se alimentando no cocho aumentou linearmente $(\mathrm{P}<0,05)$ com a elevação dos teores de concentrado, resultado previsível, uma vez que, ao se fornecerem quantidades crescentes de concentrado, os animais tendem a permanecer por maior tempo no cocho para consumir todo o suplemento. Esses resultados contrariam aqueles verificados por Bremm et al. (2005), que não notaram diferenças nos tempos que os animais permaneceram se alimentando no cocho quando receberam 0,5; 1,0 ; e $1,5 \%$ do PV em concentrado. Esses autores verificaram, no entanto, maior frequência desses animais em diferentes momentos ao longo do dia.

Os teores de concentrado não influenciaram os tempos de ruminação e outras atividades $(\mathrm{P}>0,05)$. A média de 490,63 minutos para ruminação encontrase próxima aos valores relatados por Silva et al. (2008), que observaram, também por períodos de 24 horas, vacas mestiças sob suplementação recebendo como volumoso cana-de-açúcar e encontraram tempo de 538,2 minutos de ruminação. O menor valor observado neste trabalho, comparado ao valor obtido pelos autores, se deve à menor digestão da fibra da cana-de-açúcar. Os tempos despendidos em outras atividades, neste trabalho, foi inferior aos encontrados por Silva et al. (2008), que pode ser explicado pela menor tempo de ruminação, aumentando, assim, o tempo para outras atividades.

O tempo destinado a outras atividades neste estudo encontra-se em concordância com o resultado relatado por Ferreira et al. (2005), que, avaliando vacas em lactação, relataram valores de 501 e 387 minutos para a taxa de ruminação em pastos de Brachiaria brizantha e Brachiaria decumbens. Também difere dos relatos de Pardo et al. (2003), que verificaram menores tempos de descanso para animais que não receberam suplemento. Esses mesmos autores deduzem que tal comportamento decorre do menor tempo de pastejo, em função do menor consumo de forragem pelos animais sob suplementação, sobretudo em teores mais elevados de suplementação, fato não observado neste estudo.

Não houve efeito significativo $(\mathrm{P}>0,05)$ dos teores de concentrado na dieta sobre os números de períodos de pastejo, períodos de ruminação (Tabela 6), que foram inferiores aos encontrados na literatura. $\mathrm{O}$ número de período de pastejo neste estudo esteve diretamente relacionado ao tempo de período de pastejo. Dessa forma, como o tempo de período de pastejo foi longo, houve uma diminuição direta do número de período dos animais em pastejo. Esses resultados são semelhantes aos encontrados por Cardoso et al. (2009), em pesquisa realizada com novilhas mestiças sob suplementação em pastagem de Brachiaria brizantha cv Marandu, porém diferentes dos relatados por Silva et al. (2005), que, avaliando o comportamento de novilhas durante 12 horas, verificaram efeito quadrático do aumento dos teores de suplemento na dieta sobre o número de períodos de pastejo, quando elevaram-se os teores de suplementação.

A temperatura em torno de $38^{\circ} \mathrm{C}$ durante determinados momentos do dia pode ter correlação direta com a diminuição do número de período de pastejo, uma vez que, em condições de estresse térmico, os animais diminuem a busca por forragem como forma de diminuírem o gasto energético despendido com locomoção. O número de períodos de ruminação apresentou tendência similar, e provavelmente pelo mesmo motivo, ou seja, diminuíram a ruminação como efeito do estresse térmico.

Houve efeito linear crescente dos teores de concentrado sobre o número de períodos de alimentação no cocho (NPC) e o tempo de alimentação no cocho (TPC) (Tabela 6). Animais que recebem maior teor de concentrado na dieta tendem a permanecer por mais tempo no cocho para consumir todo o concentrado, aumentando assim o número de períodos e o tempo de alimentação no cocho. Observou-se efeito quadrático do teor de concentrado também sobre o número de período em outras atividades (NPO), que foi menor entre os animais que não receberam suplemento. Os animais que receberam o maior teor de concentrado na dieta, ao terem sua exigência nutricional atendida, passaram a destinar o tempo em outras atividades, que não foram pastejo nem ruminação. 
Tabela 6. Valores médios do número de períodos de pastejo (NPP), comendo no cocho (NPC), ruminação (NNR), e ócio (NPO) e duração em (minutos) dos períodos de pastejo (TPP), comendo no cocho (TPCC), em ruminação (TPR), ócio (TPO), alimentação total (TAT) e mastigação total (TMT), com suas respectivas equações de regressão (ER), coeficientes de determinação $\left(\mathrm{R}^{2}\right)$, e coeficiente de variação $(\mathrm{CV})$.

\begin{tabular}{|c|c|c|c|c|c|c|c|}
\hline \multirow{2}{*}{ Item } & \multicolumn{4}{|c|}{ Concentrado na dieta $(\%)$} & \multirow{2}{*}{ ER } & \multirow{2}{*}{$\mathrm{R} 2$} & \multirow{2}{*}{$\mathrm{CV}$} \\
\hline & $\mathrm{C} 0,0$ & $\mathrm{C} 16,4$ & $\mathrm{C} 23,3$ & $\mathrm{C} 33,8$ & & & \\
\hline NPP & 6,94 & 8,75 & 8,13 & 7,88 & $\hat{\mathrm{Y}}=7,92$ & ----- & 23,69 \\
\hline NPR & 12,44 & 12,31 & 14,38 & 12,81 & $\hat{Y}=12,98$ & ----- & 13,21 \\
\hline NPC & 0,00 & 1,75 & 2,00 & 2,00 & 1 & 0,82 & 12,70 \\
\hline NPO & 10,69 & 13,19 & 13,50 & 12,50 & 2 & 0,99 & 13,51 \\
\hline TPP (min) & 93,37 & 81,86 & 73,33 & 81,16 & $\hat{\mathrm{Y}}=82,43$ & ----- & 35,85 \\
\hline TPC (min) & 0,00 & 14,19 & 15,94 & 20,38 & 3 & 0,95 & 35,10 \\
\hline TPR (min) & 42,46 & 39,12 & 36,98 & 36,44 & 4 & 0,95 & 16,63 \\
\hline TPO (min) & 31,30 & 24,78 & 23,39 & 28,18 & 5 & 0,99 & 19,2131 \\
\hline TAT (min) & 586,25 & 654,38 & 610,00 & 635,63 & $\hat{Y}=621,56$ & ----- & 13,66 \\
\hline TMT (min) & 1108,13 & 1115,63 & 1124,38 & 1100,63 & $\hat{Y}=1112,19$ & ----- & 5,49 \\
\hline
\end{tabular}

${ }^{1} \hat{\mathrm{Y}}=0,303952+0,06169 \mathrm{X} ;{ }^{2} \hat{\mathrm{Y}}=-0,875 \mathrm{X}^{2}+4,95 \mathrm{X}+6,6563 ;{ }^{3} \hat{\mathrm{Y}}=1,523309098+0,604173655 \mathrm{X} ;{ }^{4} \hat{\mathrm{Y}}=42,1945-0,18761 \mathrm{X} ;{ }^{5} \hat{\mathrm{Y}}=$ $43,737-15,213 \mathrm{x}+2,8277 \mathrm{x}^{2}$.

Fonte: Elaboração dos autores.

Os teores de concentrado não influenciaram o tempo por período de pastejo (TPP) (Tabela 6), embora o tempo de pastejo tenha sido longo. Essa variável estudada pode influenciada por vários fatores, como temperatura, época do ano e arquitetura e disponibilidade da forragem. Dessa forma, o longo tempo utilizado pelos animais para cada período de pastejo pode estar associado à baixa disponibilidade de forragem nos piquetes, levando aos animais a pastejar e selecionar as partes da planta com maior concentração de nutrientes.

Sarmento (2003) observou que os ruminantes tendem a ser mais seletivos em pastos com reduzida relação lâmina:colmo, o que resulta em aumento no tempo de pastejo, como mecanismo compensatório. Outra explicação pode estar relacionada ao menor valor de proteína bruta e ao maior teor de fibra em detergente neutro observados no pasto.

Houve efeito linear decrescente dos teores de concentrado sobre o tempo despendido por período de ruminação $(\mathrm{TPR})$ e efeito quadrático $(\mathrm{P}<0,05)$ sobre o tempo em outras atividades (TPO) (Tabela 6). $\mathrm{O}$ efeito linear decrescente pode ser explicado pelo fato de que os animais que receberam apenas forragem teriam um período maior de ruminação por ingerirem maior quantidade de fibra e esse tempo seria destinado à quebra da fração fibrosa da planta. Os menores tempos de período de ruminação (TPR) para os demais tratamentos estão relacionados à ingestão de carboidratos solúveis em maior quantidade presente no concentrado. Os resultados encontrados neste estudo são similares ao relatados por Cardoso (2009). Silva et al. (2005), no entanto, não verificaram efeito quando aumentaram o teor de concentrado na dieta de novilhas sob suplementação em pastagem de Brachiaria brizantha.

O tempo de período de outras atividades (TPO) está vinculado ao número de período de outras atividades (NPO). Os teores de concentrado na dieta não influenciaram o tempo de alimentação total (TAT) nem o tempo de mastigação total (TMT) (Tabela 6). O tempo de mastigação total (TMT) é obtido pela soma do tempo de alimentação e do tempo de ruminação durante as 24 horas de observação. Pereira et al. (2007) avaliaram o comportamento ingestivo de novilhas leiteiras recebendo dietas com diferentes níveis de fibra e detectaram maior tempo de mastigação total (TMT) 
quando os animais foram submetidos à dieta com teor mais elevado de FDN (60\%). Isso comprova que aspectos comportamentais dos ruminantes estão relacionados à composição química e física dos volumosos.

Os teores de concentrado não influenciaram a taxa de bocado (TxB), o tempo por deglutição (TDe) e o número de bocados dia (NBdia) (Tabela 7). Vale ressaltar que todos os animais pastejaram juntos no mesmo piquete e encontraram, portanto, um dossel forrageiro da mesma altura $(20$ a $40 \mathrm{~cm})$ e mesma quantidade de forragem disponível $(2,651 \mathrm{kgMS} /$ ha), o que pode ter contribuído para a não-diferença entre os teores de suplementação. Sarmento (2003), avaliando o comportamento ingestivo de novilhas das raças Nelore e Canchim em pasto de capimmarandu com alturas variando entre 10 e $40 \mathrm{~cm}$, observou que a taxa de bocado varia conforme a altura do dossel forrageiro.

Tabela 7. Valores médios e equações de regressão das taxas de bocados (TxB), massa de bocado, em gramas de MO de forragem por bocado $\left(\mathrm{MaB}\left(\mathrm{g}_{\mathrm{MO}}\right)\right)$, número de bocado por deglutição (BDeg), tempo de deglutição $(\mathrm{TeDeg}(\mathrm{s}))$ e número de bocados dia (NB (dia)) em função dos teores de concentrado na dieta com suas respectivas equações de regressão (ER), coeficientes de determinação $\left(\mathrm{R}^{2}\right)$, e coeficiente de variação $(\mathrm{CV})$.

\begin{tabular}{|c|c|c|c|c|c|c|c|}
\hline \multirow{2}{*}{ Item } & \multicolumn{4}{|c|}{ Concentrado na dieta $(\%)$} & \multirow{2}{*}{ ER } & \multirow{2}{*}{$\mathrm{R}^{2}$} & \multirow{2}{*}{$\mathrm{CV}$} \\
\hline & $\mathrm{C} 0,0$ & $\mathrm{C} 16,4$ & $\mathrm{C} 23,3$ & $\mathrm{C} 33,8$ & & & \\
\hline TxB (boc/s) & 0,80 & 0,83 & 0,78 & 0,78 & $\hat{\mathrm{Y}}=0,80$ & ----- & 11,70 \\
\hline $\mathrm{MaB}(\mathrm{gMO})$ & 0,40 & 0,42 & 0,55 & 0,61 & 1 & 0,82 & 19,87 \\
\hline $\mathrm{BDe}(\mathrm{n})$ & 50,94 & 40,08 & 46,74 & 41,31 & 2 & 0,48 & 23,12 \\
\hline TDeg (s) & 66,54 & 52,63 & 62,86 & 53,71 & $\hat{\mathrm{Y}}=58,93$ & ---- & 27,95 \\
\hline NB (dia) & $26.741,24$ & $30.486,33$ & $26.069,32$ & $26.322,03$ & $\hat{\mathrm{Y}}=27.404,73$ & ----- & 16,84 \\
\hline
\end{tabular}

Fonte: Elaboração dos autores.

A maior taxa de bocado $(\mathrm{TxB})$ encontrada neste estudo, quando comparada aos resultados obtidos por Zanine et al. (2007), que avaliaram taxa de bocado de novilhas no cerrado e não constataram diferenças para o total de bocados nas gramíneas Brachiaria brizantha e Brachiaria decumbens, pode ser atribuída à disponibilidade de forragem no experimento $(2,651 \mathrm{kgMS} / \mathrm{ha})$, já que o dossel forrageiro estava baixo, aumentado, assim, a taxa e o tempo de período de pastejo pelos animais.

Os teores de concentrado tiveram efeito linear crescente $(\mathrm{P}<0,05)$ sobre a massa de bocado $(\mathrm{MaB})$ e linear decrescente sobre o número de bocados por deglutição (BDe) (Tabela 7). Esse efeito crescente tratar-se de valores calculados somando-se assim os resultados de outras variáveis encontradas no presente estudo, como número de período de pastejo (NPP), tempo de período de pastejo (TPP) e taxa de bocado (TxB). O número de bocados por deglutição (BDe) foi maior no mais baixo teor de concentrado, fazendo com que os animais pudessem consumir maior quantidade de matéria seca do pasto em menor tempo, a fim de suprir suas exigências nutricionais.

Os teores de concentrado não influenciaram o número de mastigações merícicas por bolo (MMB) nem o tempo por bolo ruminado (TBo) neste estudo (Tabela 8). Esees resultados podem estar relacionados ao consumo de matéria seca do pasto, pois, mesmo com a elevação nos teores 
de concentrado, essa variável não sofreu efeito substutivo, assim, os animais precisaram mastigar e ruminar a mesma quantidade de fibra oriunda do pasto. Houve efeito linear decrescente $(\mathrm{P}<0,05)$ dos níveis de concentrado sobre o número de bolos ruminados por dia (BOL) (Tabela 8). A maior quantidade de concentrado na dieta promove rápida degradação dos carboidratos solúveis no rúmen, causando diminuição no número de bolos ruminados. $\mathrm{O}$ aumento nos teores de concentrado não influenciou a velocidade de mastigação (VeM). Houve, no entanto, efeito linear crescente sobre o tempo por mastigação (TeM), resultado que pode estar relacionado ao aumento do consumo de matéria seca. Os animais gastaram, portanto, maior tempo para quebrar a fibra do volumoso e parte do concentrado que retornou à cavidade bucal. Esse resultado foi o mesmo observado para o tempo por bolo ruminado, ou seja, de aumento conforme o nível do concentrado. Os teores de concentrado promoveram efeito linear decrescente $(\mathrm{P}<0,05)$ sobre o número de bolos ruminados por dia (BOL) (Tabela 8). Os resultados encontrados para essas variáveis também estão associados ao número de mastigações merícicas por dia, que foi maior entre os animais que não receberam concentrado. Isso ocasionou menor tempo de mastigação para compensar o menor número de bolos, e um tempo total de mastigação por dia, devido ao maior teor de fibra presente no volumoso.

Tabela 8. Valores médios dos números de MMB (mastigações merícicas por bolo); TBo - tempo por bolo ruminado; BOL - número de bolos ruminados por dia; VeM - velocidade de mastigação; TeM - tempo por mastigação; MMnd - número de mastigações merícicas por dia com suas respectivas equações de regressão (ER), coeficientes de determinação $\left(\mathrm{R}^{2}\right)$, e coeficiente de variação $(\mathrm{CV})$.

\begin{tabular}{|c|c|c|c|c|c|c|c|}
\hline \multirow{2}{*}{ Item } & \multicolumn{4}{|c|}{ Concentrado na dieta (\%) } & \multirow{2}{*}{ ER } & \multirow{2}{*}{$\mathrm{R}^{2}$} & \multirow{2}{*}{$\mathrm{CV}$} \\
\hline & $\mathrm{C} 0,0$ & $\mathrm{C} 16,4$ & $\mathrm{C} 23,3$ & $\mathrm{C} 33,8$ & & & \\
\hline $\operatorname{MMB}(n)$ & 49,29 & 47,41 & 46,99 & 49,06 & $\hat{\mathrm{Y}}=48,19$ & ----- & 8,81 \\
\hline TBo (s) & 48,22 & 47,24 & 48,03 & 50,32 & $\hat{\mathrm{Y}}=48,85$ & ----- & 9,08 \\
\hline $\mathrm{BOL}(\mathrm{n})$ & 651,96 & 586,98 & 650,36 & 556,18 & 1 & 0,45 & 16,04 \\
\hline $\operatorname{VeM}(\mathrm{n} / \mathrm{s})$ & 0,93 & 0,93 & 0,92 & 0,92 & $\hat{\mathrm{Y}}=0,93$ & ----- & 25,99 \\
\hline $\mathrm{TeM}$ & 0,98 & 1,00 & 1,02 & 1,03 & 2 & 0,92 & 4,34 \\
\hline MMnd (n) & 31691,80 & 27200,65 & 30034,78 & 26906,57 & 3 & 0,56 & 13,55 \\
\hline
\end{tabular}

${ }^{1} \hat{Y}=652,6694-2,24749 X ;{ }^{2} \hat{Y}=0,0018+0,980271 X ;{ }^{3} \hat{Y}=31183,41-121,086 \mathrm{X}$

Fonte: Elaboração dos autores.

Verificou-se crescimento linear $(\mathrm{P}<0,05)$ das eficiências de alimentação da matéria seca (EALMS), dos carboidratos não-fibrosos (EALCNF) e dos nutrientes digestíveis totais (EALNDT) com o aumento dos teores de concentrado na dieta (Tabela 9). Esses resultados podem ser explicados pelo aumento no consumo de MS total, que aumentou linearmente com os teores de concentrado. Os valores para EAL estão em concordância com o observado por
Mendonça et al. (2004), que, em pesquisa com vacas em lactação recebendo como volumoso cana-de-açúcar com dois níveis de concentrado 40 e 50\%, notaram aumento da eficiência de alimentação de acordo com o teor de concentrado. Pereira et al. (2007), por sua vez, trabalhando com diferentes teores de FDN na dieta, verificaram que a eficiência de alimentação (EAL) foi menor quando se aumentou de 30 para $60 \%$ o conteúdo de FDN na dieta. 
Tabela 9. Eficiências de alimentação e ruminação de matéria seca (MS), fibra em detergente neutro (FDN), carboidratos não-fibrosos (CNF) e nutrientes digestíveis totais (NDT), em função dos teores de concentrado na dieta, com suas respectivas equações de regressão (ER), coeficientes de determinação $\left(R^{2}\right)$, e coeficiente de variação $(C V)$.

\begin{tabular}{|c|c|c|c|c|c|c|c|}
\hline \multirow{3}{*}{ Item } & \multicolumn{4}{|c|}{ Concentrado na dieta (\%) } & \multirow{2}{*}{ ER } & \multirow{2}{*}{$\mathrm{R}^{2}$} & \multirow{2}{*}{$\mathrm{CV}$} \\
\hline & $\mathrm{C} 0,0$ & $\mathrm{C} 16,4$ & $\mathrm{C} 23,3$ & $\mathrm{C} 33,8$ & & & \\
\hline & \multicolumn{4}{|c|}{ Eficiência de alimentação } & & & \\
\hline MS (kg de MS/h) & 1,13 & 1,16 & 1,42 & 1,53 & 1 & 0,82 & 19,81 \\
\hline FDN (kg de FDN/h) & 0,72 & 0,67 & 0,79 & 0,80 & $\hat{\mathrm{Y}}=0,75$ & ---- & 20,66 \\
\hline CNF (kg de CNF/h) & 0,18 & 0,19 & 0,28 & 0,34 & 2 & 0,97 & 18,11 \\
\hline \multirow[t]{2}{*}{ NDT (kg de NDT/h) } & 0,53 & 0,61 & 0,78 & 0,89 & 3 & 0,92 & 23,27 \\
\hline & \multicolumn{4}{|c|}{ Eficiência de ruminação } & & & \\
\hline MS (kg de MS/h) & 1,26 & 1,71 & 1,76 & 2,10 & 4 & 0,98 & 17,98 \\
\hline FDN (kg de FDN/h) & 0,79 & 0,89 & 0,92 & 1,09 & 5 & 0,92 & 18,20 \\
\hline $\mathrm{CNF}(\mathrm{kg}$ de $\mathrm{CNF} / \mathrm{h}$ ) & 0,19 & 0,29 & 0,32 & 0,46 & 6 & 0,94 & 18,75 \\
\hline NDT (kg de NDT/h) & 0,57 & 0,75 & 0,90 & 1,21 & 7 & 0,93 & 19,19 \\
\hline
\end{tabular}

${ }^{1} \hat{Y}=1,07445+0,012729 \mathrm{X} ;{ }^{2} \hat{Y}=0,157263+0,004822 X ;{ }^{3} \hat{Y}=0,497767+0,011152 X ;{ }^{4} \hat{Y}=1,262737+0,024208 X ;{ }^{5} \hat{Y}=$ $0,767299+0,008537 \mathrm{X} ;{ }^{6} \hat{\mathrm{Y}}=0,176607+0,007582 \mathrm{X} ;{ }^{7} \hat{\mathrm{Y}}=0,519472+0,018403 \mathrm{X}$

Fonte: Elaboração dos autores.

Os teores de concentrado na dieta de vacas mestiças não influenciaram $(\mathrm{P}>0,05)$ a eficiência de alimentação da fibra em detergente neutro (EALFDN), mesmo com o aumento linear no consumo de FDN na dieta total, o que pode ser atribuído à qualidade da fibra presente no concentrado. Bürger et al. (2000) encontraram efeito quadrático, diferente do observado por Mendonça et al. (2004), que não encontraram efeito, como observado neste estudo.

A eficiência de ruminação é importante no controle da utilização de volumosos e, quando ocorre uma redução nesta eficiência, essa redução não pode ser compensada pelo prolongamento da atividade de ruminação. A eficiência de ruminação da dieta, expressa em kg/MS.h (ER), kg FDN/h $\left(\mathrm{ER}_{\mathrm{FDN}}\right), \mathrm{kg} \mathrm{CNF} / \mathrm{h}\left(\mathrm{ER}_{\mathrm{CNF}}\right)$ e $\mathrm{kg} \mathrm{NDT/h}\left(\mathrm{ER}_{\mathrm{NDT}}\right)$ aumentou linearmente $(\mathrm{P}<0,05)$. Segundo Dulphy, Remond e Theriez (1980), a eficiência de ruminação aumenta com a elevação do teor de concentrado na dieta. Esse fato também foi verificado por Bürger et al. (2000), que relataram aumento linear da eficiência de ruminação (ERU) com a inclusão de concentrado nas dietas, enquanto a $E R U_{F D N}$ decresceu linearmente. Os autores explicaram que esses resultados foram provocados pela baixa degradação da fibra do volumoso e pela baixa atividade dos microrganismos celulolíticos, além da regurgitação de parte do concentrado no bolo durante a ruminação.

\section{Conclusões}

A suplementação com até $33,8 \%$ de concentrado na dieta de vacas mestiças em pastejo não influencia nas atividades de pastejo e ruminação e em outras atividades, porém aumenta o tempo despendido em alimentação no cocho.

O aumento no teor do concentrado da dieta melhora a eficiência de alimentação da matéria seca, dos carboidratos não-fibrosos e nutrientes digestíveis totais, além da eficiência de ruminação.

\section{Referências}

BAGGiO, C.; CARVALHO, P. C. F.; SILVA, J. L. S.; ANGHINONI, I.; LOPES, M. L.T.; THUROW, J. M. Padrões de deslocamento e captura de forragem por novilhos em pastagem de azevém-anual e aveia-preta manejada sob diferentes alturas em sistema de integração lavoura-pecuária. Revista Brasileira de Zootecnia, Viçosa, MG, v. 38, n. 2, p. 215-222, 2009. 
BREMM, C.; ROCHA, M. G.; RESTLE, J.; PILAU, A.; MONTAGNER, D. B.; FREITAS, F. K.; MACARI, S.; ELEJALDE, D. A.; ROSO, G. D.; ROMAN, J.; GUTERRES, É. P.; COSTA, V. G.; NEVES, F. P. Efeito de níveis de suplementação sobre o comportamento ingestivo de bezerras em pastagem de aveia (Avena strigosa Schreb.) e Azevém (Lolium multiflorum Lam.). Revista Brasileira de Zootecnia, Viçosa, MG, v. 34, n. 2, p. 387-397, 2005.

BÜRGER, P. J.; PEREIRA, J. C.; QUEIROZ, A. C.; SILVA, J. F. C.; VALADARES FILHO, S. C.; CECON, P. R.; CASALI, A. D. P. Comportamento ingestivo em bezerros holandeses alimentados com dietas contendo diferentes níveis de concentrado. Revista Brasileira de Zootecnia, Viçosa, MG, v. 29, n. 1, p. 236-242, 2000.

CARDOSO, E. O.; SANTANA JUNIOR, H. A.; SILVA R. R.; PEREIRA, M. M.; LACERDA, J .J. D.; ABREU FILHO, G.; BARROSO, D. S. Discretização de séries temporais no comportamento ingestivo de novilhas leiteiras sobre manejo nutricional visando ganho compensatório. In: REUNIÃO ANUAL DA SOCIEDADE BRASILEIRA DE ZOOTECNIA, 46., 2009, Maringá. Anais... Maringá: SBZ, 2009. CD-ROM.

DULPHY, J. P.; REMOND, B.; THERIEZ, M. Ingestive behavior and related activities in ruminants. In: RUCKEBUSH, Y.; THIVEND, P. (Ed.). Digestive physiology and metabolism in ruminants. Lancaster: MTP, 1980. p. 103-122.

FERREIRA, D. J.; ZANINE, A. M.; SANTOS, E. M.; PARENTE, H. N.; MACEDO JÚNIOR, G. L.; CECON, P. R. Comportamento ingestivo de vacas lactantes em pastagens de Brachiaria brizantha e Brachiaria decumbens. In: REUNIÃO ANUAL DA SOCIEDADE BRASILEIRA DE ZOOTECNIA, 42., 2005, Goiânia. Anais... Goiânia: SBZ, 2005. CD-ROM.

FISCHER, V.; DESWYSEN, A. G.; DUTILLEUL, P.; BOEVER, J.; Padrões da distribuição nictemeral do comportamento ingestivo de vacas leiteiras, ao inicio e ao final da lactação, alimentadas com dieta à base de silagem de milho. Revista Brasileira de Zootecnia, Viçosa, MG, v. 31, n. 5, p. 2129-2138, 2002.

GARDNER, A. L. Técnicas de pesquisa em pastagem e aplicabilidade de resultados em sistema de produção. Brasília: IICA/EMBRAPA CNPGL, 1986. 197 p.

HALL, M. B. Challenges with non-fiber carbohydrate methods. Journal of Animal Science, Champaign, v. 81, n. 12, p. 3226-3232, 2003.

HANCOCK, J. Grazing behaviour of cattle. Animal Breeding Abstract, Wallingford, v. 21, n. 1, p. 1-13, 1953.

HODGSON, J. Ingestive behavior. In: LEAVER, J.
D. (Ed.). Herbage intake handbook. Hurley: British Grassland Society, 1982. p. 113-138.

JAMIESON, W. S.; HODGSON, J. The effect of variation in sward characteristics upon the ingestive behavior and herbage intake of calves and lambs under continuous stocking management. Grass and Forage Science, Chichester, v. 34, n. 4, p. 273-281, 1979.

McMENIMAN, N. P. Methods of estimating intake of grazing animals. In: REUNIÃO ANUAL DA SOCIEDADE BRASILEIRA DE ZOOTECNIA, 34., 1997, Juiz de Fora. Anais... Juiz de Fora: SBZ, 1997. CD-ROM.

MENDES NETO, J.; CAMPOS, J. M. S.; VALADARES FILHO, S. C.; LANA, R. P.; QUEIROZ, A. C.; EUCLYDES, R. F. Comportamento ingestivo de novilhas leiteiras alimentadas com polpa cítrica em substituição ao feno de capim-tifton 85. Revista Brasileira de Zootecnia, Viçosa, MG, v. 36, n. 3, p. 618-625, 2007.

MENDONÇA, S. S.; CAMPOS, J. M. S.; VALADARES FILHO, S. C.; VALADARES, R. F. D.; SOARES, C. A.; LANA, R. P.; QUEIROZ, A. C.; ASSIS, J.; PEREIRA, M. L. A. Comportamento ingestivo de vacas leiteiras alimentadas com dietas à base de cana-de-açúcar ou silagem de milho. Revista Brasileira de Zootecnia, Viçosa, MG, v. 33, n. 3, p. 723-728, 2004.

MERTENS, D. R. Gravimetric determination of amylasetreated neutral detergent fiber in feeds with refluxing in beaker or crucibles: collaborative study. Journal of $A O A C$ International, Madinson, v. 85, n. 6, p. 1217-1240, 2002.

MINSON, D. J. Forage in ruminant nutrition. San Diego: Academic Press, 1990. 483 p.

MORENO, C. B. Efeito da suplementação com farelo de milho sobre o desenvolvimento corporal de novilhas leiteiras sobre pastagem de azevém (Lolium multiflorum). In: REUNIÃO ANUAL DA SOCIEDADE BRASILEIRA ZOOTECNIA, 39., 2002, Recife. Anais... Recife: SBZ, 2002. CD-ROM.

NATIONAL RESEARCH COUNCIL - NRC. Nutrient requirements of dairy cattle. 7. ed. Washington, D.C.: National Academy Press, 2001. 381 p.

PARDO, R. M. P.; FISCHER, V.; BALBINOTTI, M.; MORENO, C. B.; FERREIRA, E. X.; VINHAS, R. I.; MONKS, P. L. Comportamento ingestivo diurno de novilhos em pastejo submetidos a níveis crescentes de suplementação energética. Revista Brasileira de Zootecnia, Viçosa, MG, v. 32, n. 6, p. 1408-1418, 2003.

PAULINO, M. F.; DETMANN, E.; VALADARES FILHO, S. C. Suplementação animal em pasto: energética ou protéica? In: SIMPÓSIO SOBRE MANEJO 
ESTRATÉGICO DA PASTAGEM, 3., 2006, Viçosa, MG. Anais... Viçosa, MG: SIMFOR, 2006. p. 359-392.

PEREIRA, J. C.; CUNHA, D. N. F. V.; CECON, P. R.; FARIA, E. S. Comportamento ingestivo e taxa de passagem de partículas em novilhas leiteiras de diferentes grupos genéticos submetidas a dietas com diferentes níveis de fibra. Revista Brasileira de Zootecnia, Viçosa, MG, v. 36, n. 6, p. 2134-2142, 2007.

PRACHE, S.; GORDON, I. J.; ROOK, A. J. Foraging behaviour and diet selection in domestic herbivores. Annales de Zootechnie, Paris, v. 48, n. 1, p. 1-11, 1998.

RIBEIRO JÚNIOR, J. I. Análises estatísticas no SAEG: sistema de análises estatísticas. Viçosa: Universidade Federal de Viçosa, MG, 2001. 301 p.

RUTTER, S. M.; ORR, R. J.; PENNING, P. D.; YARROW, N. H. Ingestive behaviour of heifers grazing monocultures of ryegrass or white clover. Applied Animal Behaviour Science, Boston, v. 76, n. 1, p. 1-9, 2002.

SARMENTO, D. O. L. Comportamento ingestivo de bovinos em pastos de capim-Marandu, submetidos a regimes de lotação contínua. 2003. Dissertação (Mestrado em Zootecnia) - Escola Superior de Agricultura Luiz de Queiroz. Universidade de São Paulo, Piracicaba.

SILVA, D. J.; QUEIROZ, A. C. Análise de alimentos: métodos químicos e biológicos. Viçosa: Universidade Federal de Viçosa, MG, 2002. 235 p.

SILVA, F. F., SÁ, J. F., SCHIO, A. R.; ÍTAVO L. C. V.; SILVA, R. R.; MATEUS, R. G. Suplementação a pasto: disponibilidade e qualidade $\mathrm{x}$ níveis de suplementação $\mathrm{x}$ desempenho, Revista Brasileira de Zootecnia, Viçosa, MG, v. 38, p. 371-389, 2009. Suplemento especial.

SIlVA, R. R.; SILVA, F. F.; CARVALHO, G. G. P.; VELOSO, C. M.; FRANCO, I. L.; AGUIAR, M. S. M. A.; CHAVES, M. A.; CARDOSO, C. P.; SIlVA, R. R. Avaliação do comportamento ingestivo de novilhas $3 / 4$ Holandês x Zebu alimentadas com silagem de capimelefante acrescida de $10 \%$ de farelo de mandioca: aspectos metodológicos. Ciência Animal Brasileira, Goiânia, v. 6, n. 3, p. 173-177, jul./set. 2005.

SILVA, R. R.; PRADO, I. N.; CARVALHO, G. G. P.; SANTANA JUNIOR, H. A.; SILVA, F. F.; DIAS, D. L. S. Efeito da utilização de três intervalos de observações sobre a precisão dos resultados obtidos no estudo do comportamento ingestivo de vacas leiteiras em pastejo. Ciência Animal Brasileira, Goiânia, v. 9, n. 2, p. 319326, abr./jun. 2008.

SILVA, R. R. Terminação de novilhos nelore suplementados em pastagens: comportamento, desempenho, características da carcaça e da carne e a economicidade do sistema. 2008. Tese (Doutorado em Zootecnia) - Universidade Estadual de Maringá, Maringá.

SILVA, R. R.; MAGALHÃES, A. F.; CARVALHO, G. G. P.; SILVA, F. F.; FRANCO, I. L.; NASCIMENTO, P. V. N.; BONOMO, P. Comportamento ingestivo de novilhas mestiças de holandês suplementadas em pastejo de Brachiaria. Aspectos metodológicos. Revista Eletrônica de Veterinária, Andalucía, v. 5, n. 10, p. 1-10, 2004.

SOUSA, B. M.; SATURNINO, H. M.; BORGES, A. L. C. C.; LOPES, F. C. F.; SILVA, R. R.; CAMPOS, M. M.; PIMENTA, M.; CAMPOS, W. E. Estimativa de consumo de matéria seca e de fibra em detergente neutro por vacas leiteiras sob pastejo, suplementadas com diferentes quantidades de alimento concentrado. Arquivo Brasileiro Medicina Veterinária e Zootecnia, Belo Horizonte, v. 60, n. 4, p. 890-895, 2008.

WILM, H. G.; COSTEllo, D. F.; KLIPPLE, G. E. Estimating forage yield by the double sampling method. Journal of American Society of Agronomy, Washington, v. 36, n. 3, p. 194-203, 1994.

ZANINE, A. M.; SANTOS, E. M.; PARENTE, H. N.; FERREIRA, D. J.; OLIVEIRA, J. S.; LANA, R. P. MACEDO JÚNIOR, G. L. Habito de pastejo de novilhas em pastagens do gênero Brachiaria. Acta Scientiarum Animal Sciences, Maringá, v. 29, n. 4, p. 365-369, 2007. 